\title{
A Radiation Tolerant Video Camera for High Total Dose Environments
}

\author{
Gordon R. Hopkinson, Member, IEEE, Mark D Skipper and Barry Taylor \\ Sira Electro-Optics Ltd, \\ South Hill, Chislehurst, Kent BR7 5EH, UK \\ e-mail: gordon.hopkinson@siraeo.co.uk).
}

\begin{abstract}
The design and performance of a solid state video camera, tolerant to $>5.3 \mathrm{Mrd}(\mathrm{Si})$, is described. The camera uses a commercially available CMOS active pixel sensor (APS) and is suitable for high total dose applications in the nuclear industry.
\end{abstract}

Keywords-APS; CMOS; imager; nuclear; camera; video; sensor; radiation

\section{INTRODUCTION}

In the nuclear power industry there are many applications where the robustness and reliable pixel geometry of a solid state video camera are needed but tolerance to a high gamma dose is also required. Examples are in-core metrology and process line inspection. This paper discusses the design and performance of a camera based on a commercially available radiation hardened CMOS active pixel sensor (APS), the star250 device from FillFactory NV, Belgium. Eid et al. [1] have also discussed the performance of a radiation-hardened APS. Previously, charge injection devices (CIDs) have been the only solid state imagers with Mrd tolerance levels ([2],[3]).

The camera has a split-box configuration where only the camera head (CMOS APS and proximity electrons) needs to be exposed to the radiation environment. The camera head has been shown to withstand 5.3 $\mathrm{Mrd}(\mathrm{Si})$ cobalt60 irradiation whilst powered and operating (the control unit and lens were not irradiated).

Images were also taken during the irradiation so as to assess the noise from gamma 'events'. It was found that useable imaging could be obtained at a dose rate of $20 \mathrm{krd}$ $(\mathrm{Si}) / \mathrm{hr}$ and even at $200 \mathrm{krd} / \mathrm{hr}$ discernible images could be obtained, though at reduced signal-to-noise ratio.

\section{EXPERIMENTAL}

\section{A. Camera System Testing}

The camera head uses the Star-250 512 x 512 pixel APS from FillFactory, NV, Belgium [4]. This is a radiation hardened version of the sensor previously tested in reference [5]. The pixel size is $25 \mu \mathrm{m} \times 25 \mu \mathrm{m}$. The electro-optical performance is similar to the previous version but the quantum efficiency in the red is reduced because of the use of a thin (4 $\mu \mathrm{m}$ ) epitaxial layer and the crosstalk (and MTF) is improved through the use of four photodiodes per pixel. The manufacturer achieved hardening by design (through use of enclosed, gate-all-around NMOS transistors) and has previously verified performance for pixel test structures [6]. There was no adjustment of operating voltages during the testing, indicating that threshold voltage shifts in the thin gate oxides were not significant. This was verified during later tests in the laboratory.

A block diagram of the camera is shown in Fig. 1. The head contains only the APS device, biasing resistors, decoupling capacitors and a radiation-tolerant, high band width video amplifier for driving the analog signal down the $12.5 \mathrm{~m}$ of connecting cable. With this length of cable there was no discernible degradation in video signal. With a lower capacitance cable it is expected that a longer length could be used $(30-50 \mathrm{~m}$ is commonly needed in nuclear installations). Although there is an on-chip 10-bit ADC this was not used in the video camera.

The control unit included a Xilinx FPGA for generating the clocks and command signals for the APS. This chip also provided signals to convert the APS output waveform (which is not CCIR standard) to a form suitable for display on a CCIR TV screen (provision of sync. pulse during line blanking and 2:1 interlace). The APS output has 4 programmable gains, which could be set from the controller. Also included in the controller were simple line drivers (with resistor termination at the camera head end), a video amplifier for adding the sync. pulse to the waveform and a power supply module. The use of a re-progammable Xilinx FPGA allows for easy customization to suite a variety of display formats (e.g. US RS170 TV, as well as European CCIR, formats). Windowed readout of selected regions of interest is also possible.

The head itself was made as small as possible, given the size of the image sensor, and measured only $53 \mathrm{~mm}$ in diameter $x 35 \mathrm{~mm}$ length. A photograph is shown in Fig. 2.

Images were captured onto a standard video recorder. Selected frames were saved as 8-bit tiff files. 


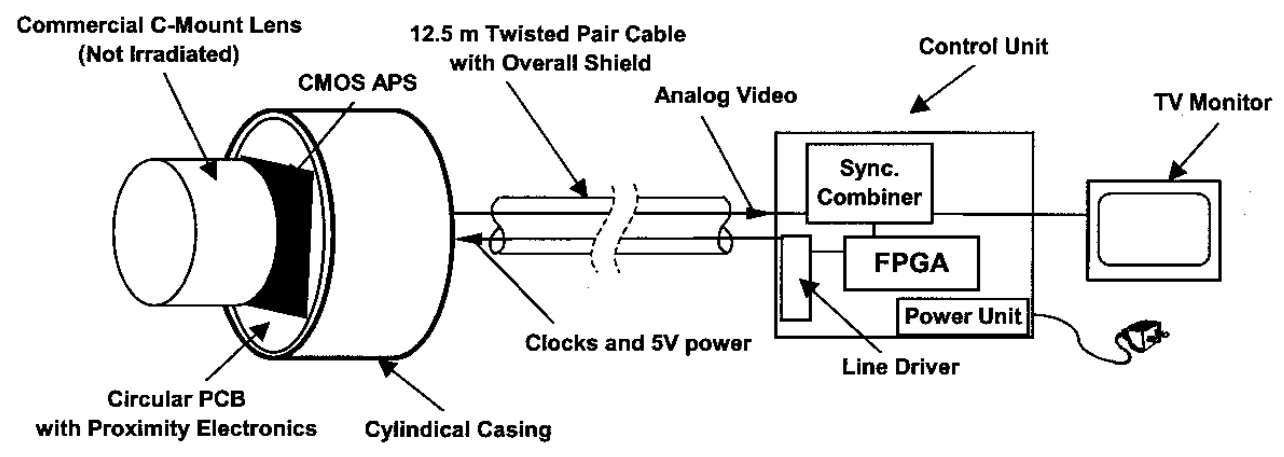

Fig. 1. Block diagram of the camera showing the split box configuration and connecting cable

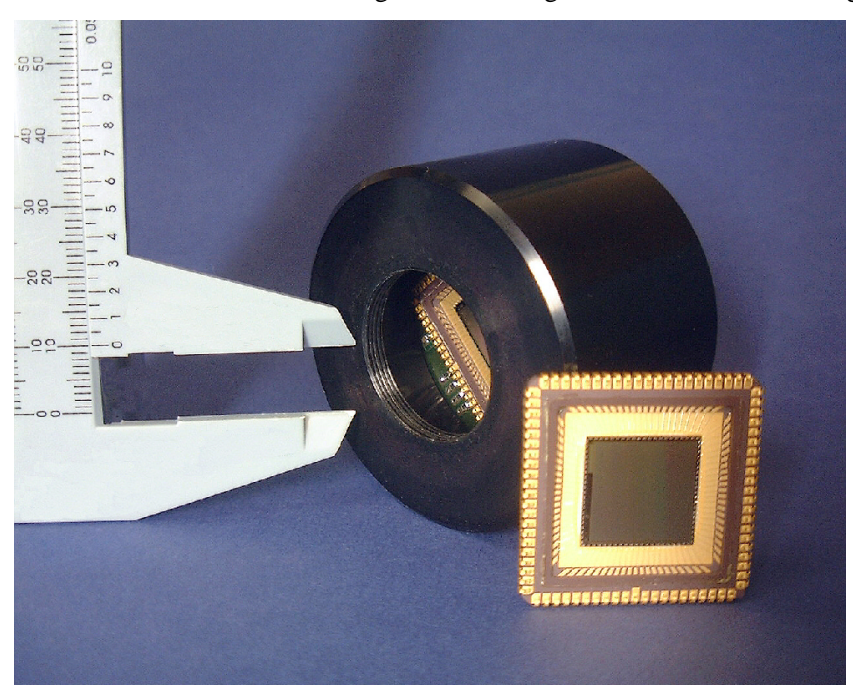

Fig. 2. Photograph of the camera head and APS chip

\section{B. Laboratory APS Testing}

After completion of the irradiation and video camera tests, the APS was de-soldered from the camera head circuit board and characterized with a laboratory detector evaluation system. This allowed determination of clock threshold voltages and ADC differential nonlinearity (DNL) as well as examination of images digitized either with the on-chip ADC or with an external ADC (choice of either 14- or 16-bit). For these tests a pixel rate of $5 \mathrm{MHz}$ was used. Measurements were made of room temperature dark current and fixed pattern noise, full well capacity, power consumption and relative responsivity. All measurements were made for both the irradiated device and four nonirradiated devices from the same wafer lot. The interval between irradiation and laboratory tests was 5.5 months, during which the sensor was kept unbiased at room temperature.

\section{Irradiations}

The cobalt60 gamma irradiation was performed at room temperature at the J. J. Thomson Irradiation Laboratory of Cranfield University, Shrivenham, UK. The initial dose rate was $20 \mathrm{krd}\left(\mathrm{H}_{2} \mathrm{O}\right) / \mathrm{hr}$ (for the first $43.8 \mathrm{hr}$ ) increasing to 100 $\mathrm{krd}\left(\mathrm{H}_{2} \mathrm{O}\right) / \mathrm{hr}$ for the next $45.5 \mathrm{hr}$ and finally $200 \mathrm{krd}\left(\mathrm{H}_{2} \mathrm{O}\right) / \mathrm{hr}$ for the final $2.4 \mathrm{hrs}$ - so that the total accumulated dose was
5.3 $\mathrm{Mrd}(\mathrm{Si})$. Dosimetry is believed to be accurate to better than $10 \%$. Note that $1 \mathrm{rd}\left(\mathrm{H}_{2} 0\right)=0.896 \mathrm{rd}(\mathrm{Si})$.

The camera head was biased and clocked throughout, but the on-chip ADC was not used and was kept unbiased. For checking of picture quality after each dose step a commercial C-mount lens was used, together with a standard test chart. However the lens was found to darken significantly in the irradiation beam (though it subsequently annealed) and so for measurements during irradiation (at the two highest dose rates: 100 and $\left.200 \mathrm{krd}\left(\mathrm{H}_{2} \mathrm{O}\right) / \mathrm{hr}\right)$ a simple aperture was placed in front of the sensor to form a 'pinhole' camera. This was used to form images of a pair of fluorescent light tubes mounted on the ceiling.

\section{RESULTS}

\section{A. Camera Tests}

All the tests were performed with the camera and APS at room temperature (the sensor was not cooled). Figs 3 and 4 show test chart images taken before and after irradiation (to 5 $\operatorname{Mrd}(\mathrm{Si}))$. It can be seen that image quality was not significantly diminished and the camera remained fully functional with good imaging performance. An increase in surface dark current was noticed but this showed some annealing after irradiation.

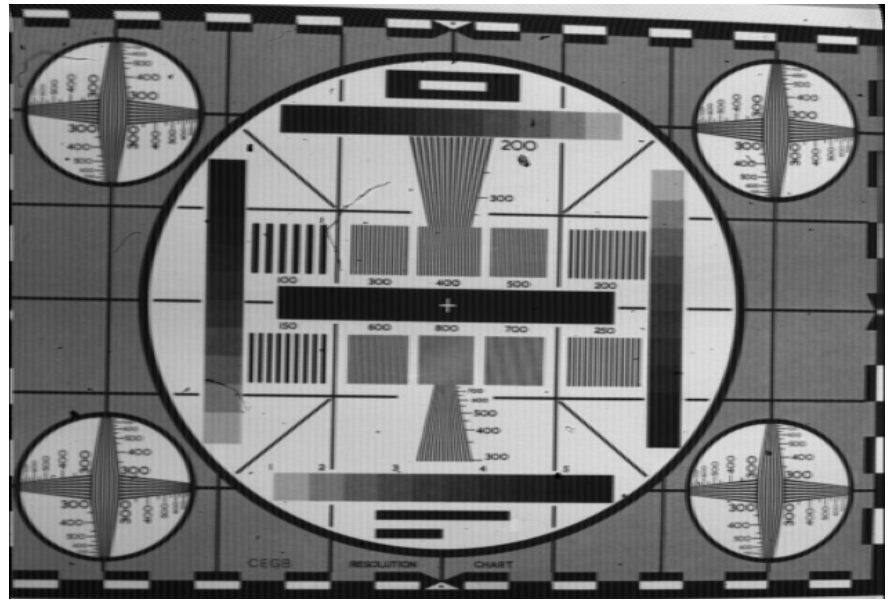

Fig. 3. Test chart image before irradiation 


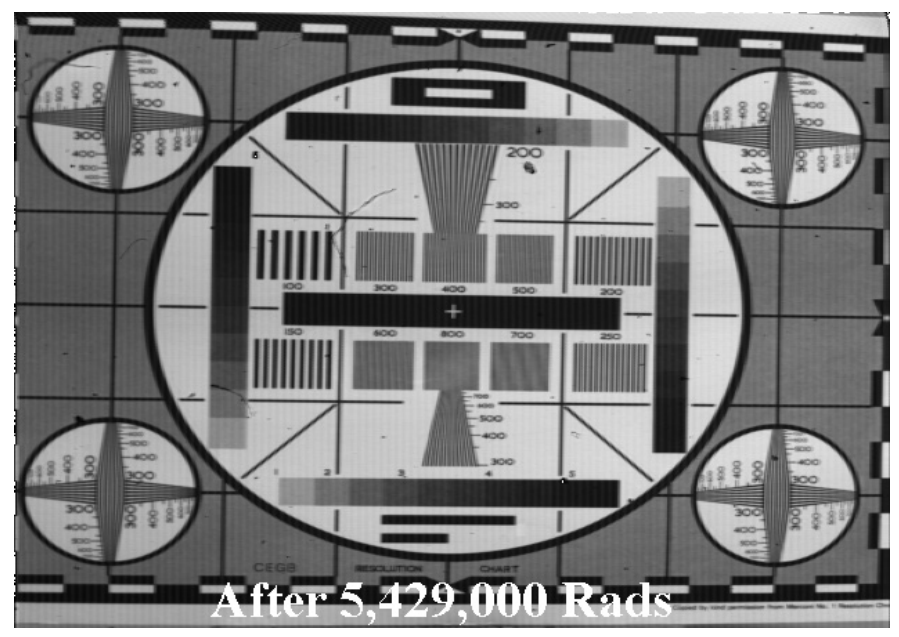

Fig. 4. Test chart image after $5 \mathrm{Mrad}(\mathrm{Si})$, note the image annotation is in $\operatorname{rads}\left(\mathrm{H}_{2} \mathrm{O}\right)$

Figs. 5, 6 and 7 show pinhole camera images without irradiation, during irradiation at $100 \mathrm{krd} / \mathrm{hr}$ and during 200 $\mathrm{krd} / \mathrm{hr}$, respectively (the position of the camera was shifted between taking Figs 6 and 7 so the fluorescent lights appear to have moved). These are single frames, taken at 50 frames per second and there was no frame averaging. Although the images are derived from a video tape recording and the lighting conditions were not accurately controlled, it is possible to make qualitative judgments of the imaging performance. It can be seen that at $100 \mathrm{krd} / \mathrm{hr}$ the image quality is degraded by gamma events generated in the silicon (giving an increased baseline signal and increased noise) but the image quality would be useful for many applications. At $200 \mathrm{krd} / \mathrm{hr}$ the image quality is significantly degraded but the fluorescent lights can still be discerned. Fig. 8 shows a horizontal scan through the three images (with the profile for the final image shifted so that the peaks due to the lights are aligned). The increase in baseline and noise with dose rate can clearly be seen. Note that at the highest dose rate the peak signals are saturated. Pulse height distributions are given in Fig. 9.

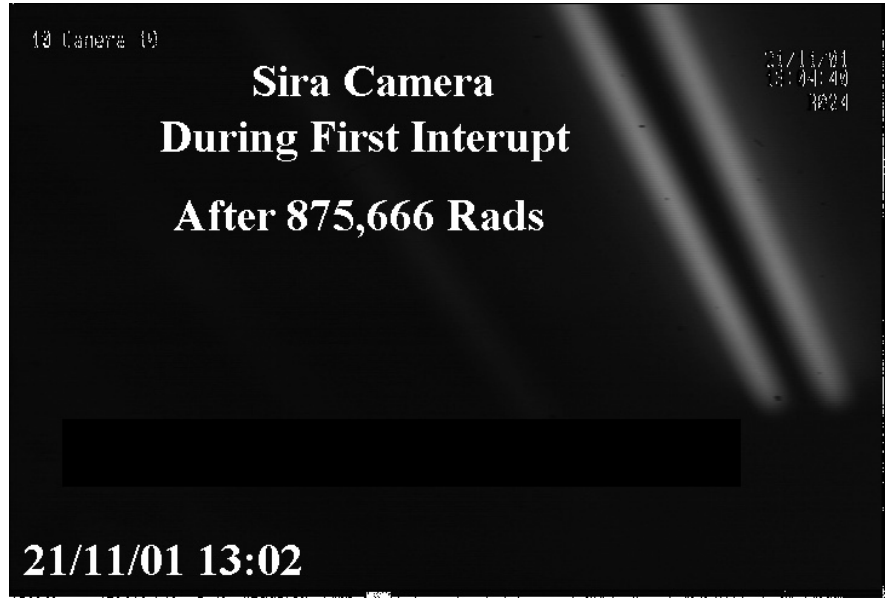

Fig. 5 Pinhole camera image at $0 \mathrm{krd} / \mathrm{hr}$ dose rate (i.e. beam not on).

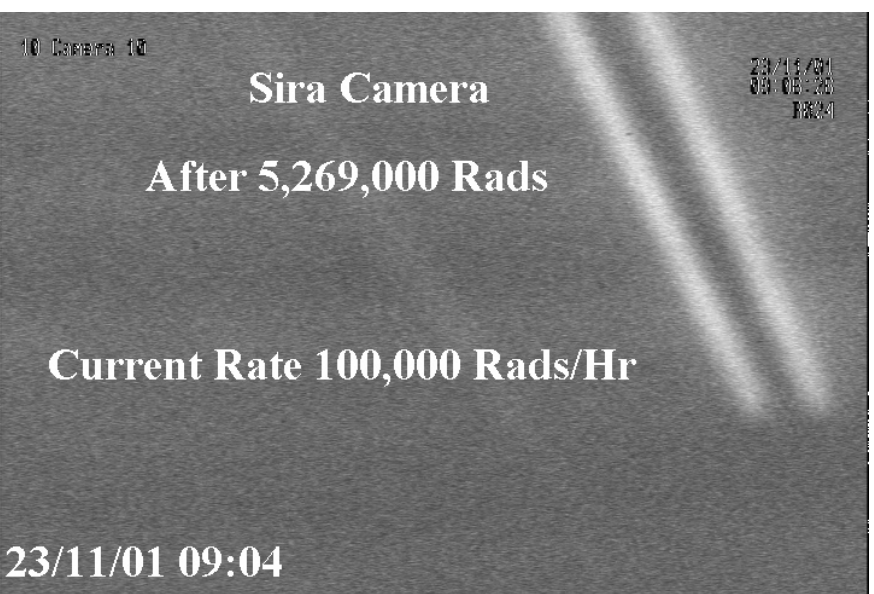

Fig. 6 Pinhole camera image at $100 \mathrm{krd}\left(\mathrm{H}_{2} \mathrm{O}\right) / / \mathrm{hr}$ dose rate.

\section{Sira Camera}

After 5,892,333 Rads

\section{Current Rate 200,000 Rads/r}

\section{3/11/01 14:29}

Fig. 7 Pinhole camera image at $200 \mathrm{krd}\left(\mathrm{H}_{2} 0\right) / / \mathrm{hr}$ dose rate.

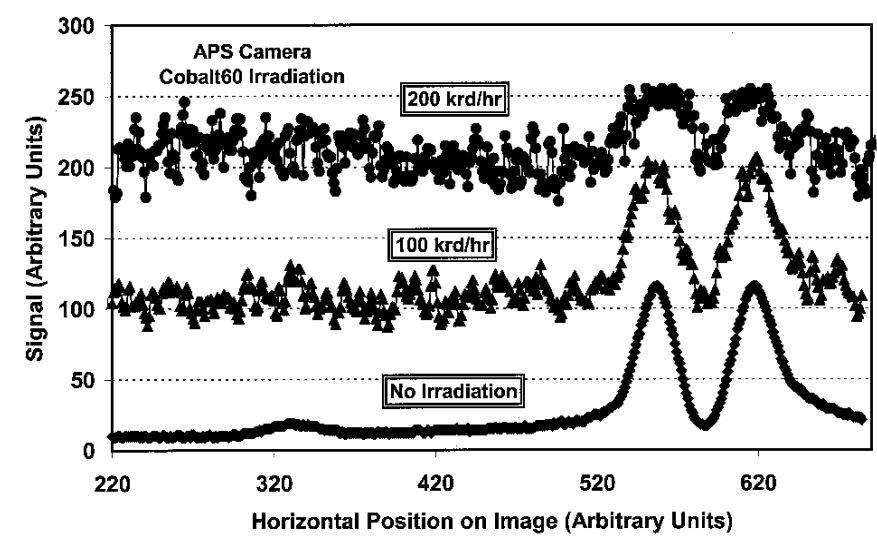

Fig. 8 Horizontal profiles through Figs 5, 6 and 7 (the profile for Fog. 7 was shifted so that the peaks were aligned). The peaks for the $200 \mathrm{krd}\left(\mathrm{H}_{2} 0\right) / \mathrm{hr}$ image are saturated. 


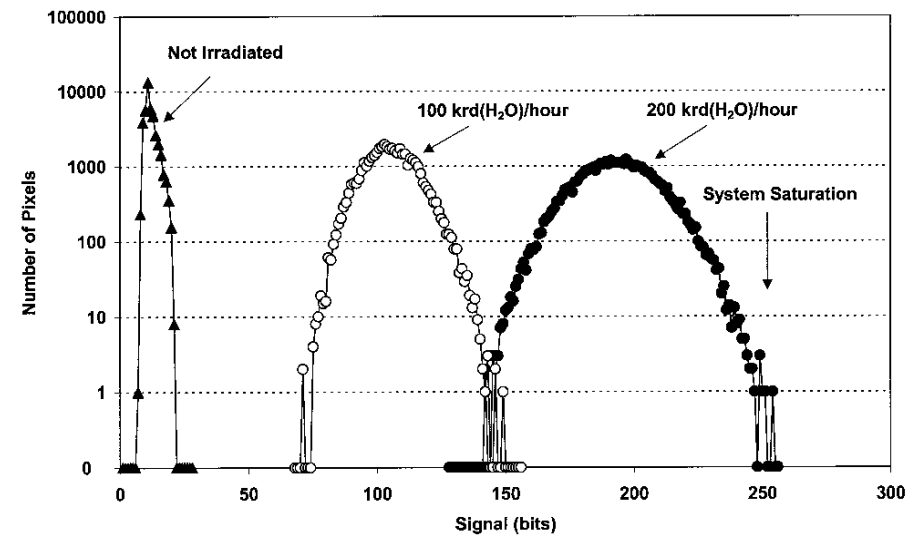

Fig. 9 Pulse height distributions for images recorded during the dose rate tests

These 'dose rate' images represent a reasonably worst case since there is no temporal averaging (which is very effective in removing the 'speckle' from gamma ray events), also the black level (baseline) increase has not been compensated. Figures 10 and 11 show test card images where the baseline has been removed and the contrast 'stretched'. These are still single frame images however.

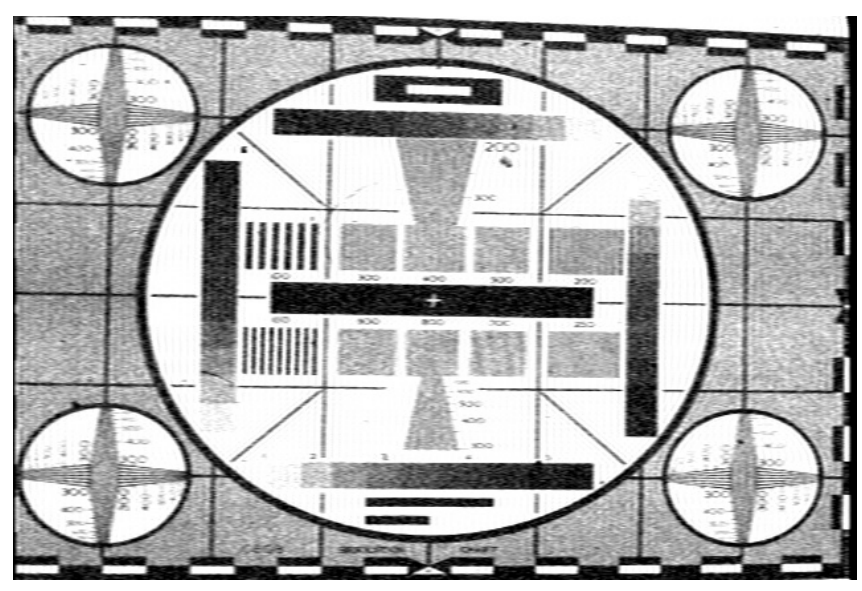

Fig. 10 Test chart image at $100 \mathrm{krd}\left(\mathrm{H}_{2} \mathrm{O}\right) / \mathrm{hr}$ dose rate., after $5.3 \mathrm{Mrd}(\mathrm{Si})$ total accumulated dose.

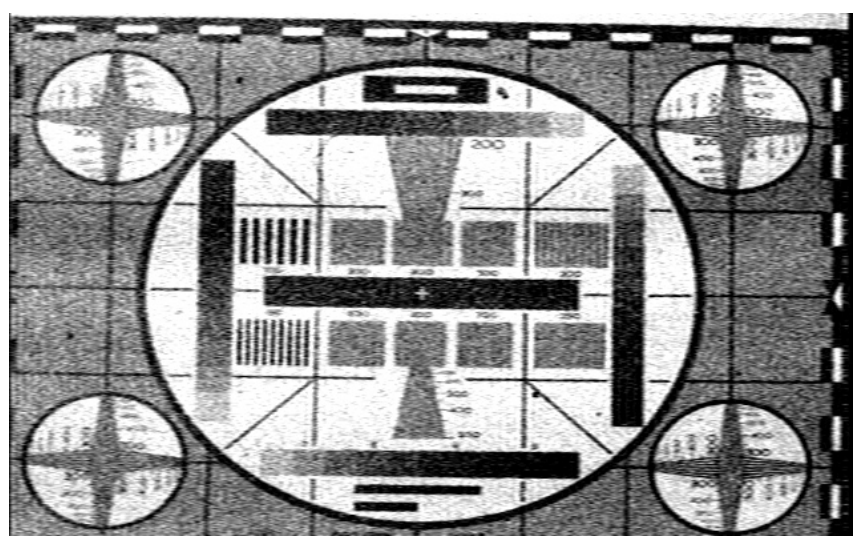

Fig. 11 Test chart image at $200 \mathrm{krd}\left(\mathrm{H}_{2} \mathrm{O}\right) / \mathrm{hr}$ dose rate., after $5.3 \mathrm{Mrd}(\mathrm{Si})$ total accumulated dose.

\section{B. Laboratory Tests}

Fig. 12 shows the voltage thresholds for the clock high and low levels beyond which the device did not function. (If the clock high voltage was below threshold or the clock low voltage was above threshold then the sensor gave either no or abnormal images.) These thresholds were obtained by varying the clock voltages under computer control and monitoring the digitized pixel signals (using the internal ADC and electronic shuttering to reduce the integration time). The 10 clock signals which are needed to drive the ADC and the row, column and reset registers are labeled in the diagram and explained in the manufacturer's data sheet [4]. In all cases there was a sharp transition between functional and nonfunctional conditions for the device. The figure also shows threshold voltages for four un-irradiated devices from the same wafer lot. The error bars show the spread in the threshold values for these four devices. The 5.3 Mrd thresholds are consistently below the unirradiated values with an average difference of $80 \mathrm{mV}$ with a standard deviation of the mean $20 \mathrm{mV}$. (So the average threshold shift after 5.3 Mrd was $80 \pm 20 \mathrm{mV}$.) Note that a much larger threshold voltage swing could be tolerated before the device fails to operate (typical applied clock swings are 0 to $3.3 \mathrm{~V}$ ).

For signal measurements the calibration of ADC units in terms of electrons/pixel was made by measuring the full well capacity (saturation) of the four un-irradiated devices and equating this to the manufacturer's average value of 310,000 electrons. This calibration should be accurate to around $15 \%$. With this value, the post-irradiation dark current at room temperature $\left(21 \pm 2^{\circ} \mathrm{C}\right)$ was 310,000 electrons/pixel/s (that this is the same number as for full well capacity is coincidental). Compare this with the average dark current of the unirradiated devices of 1,600 electrons/pixel/s, which is low because of the small depleted volume of the photodiodes within the pixel (and is comparable with un-irradiated inverted mode CCDs).

= Average HI, no irrad. $\square 5$ Mrad HI $=$ Average LO, no irrad. $\square 5$ Mrad LO

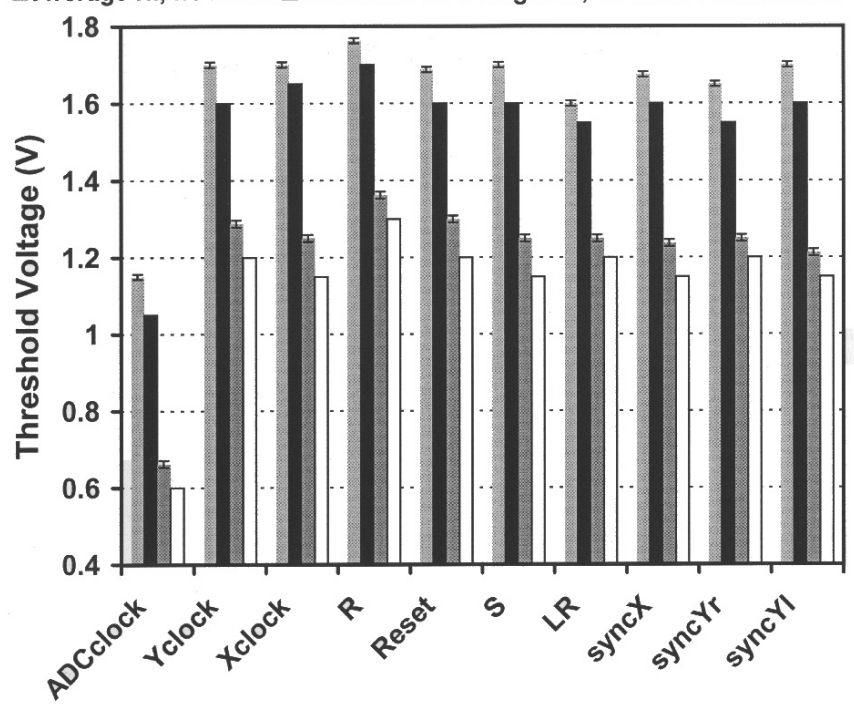

Fig 12 Clock threshold voltages for un-irradiated (gray) and irradiated (black and white) devices. The error bars show the spread in values for the four unirradiated sensors. 
At TV rate the post-irradiation dark current is not large enough to give an obvious reduction in dynamic range but it does give a 'grainy' appearance to the images when they are examined closely.

Electronic fixed pattern noise, FPN (i.e. the pixel-to-pixel variations in offset) was similar for both the irradiated device and the unirradiated devices and it is concluded that the irradiation had no significant effect on FPN.

The current drawn from the digital $5 \mathrm{~V}$ supply to the ADC was $96 \mathrm{~mA}$ for the irradiated device, which is little different from the unirradiated devices (average $93 \mathrm{~mA}$ with a spread of $\pm 3 \mathrm{~mA}$ ). All the control devices had an analog 5V supply current of $23 \mathrm{~mA}$ which decreased to $18 \mathrm{~mA}$ for the $5.3 \mathrm{Mrd}$ device. So the irradiation caused a negligible change in power consumption. (Note that the ADC supply current can be reduced for slow pixel rate applications as it is strongly dependent on clock frequency.)

In most remote head nuclear applications (where signals need to be sent down long lengths of cable) the internal ADC will not be used since the analog output is preferable to implementing a high speed radiation tolerant serial link. (If necessary, the analog signal can be digitized in the controller.) This makes the radiation tolerance of the ADC of 'academic' interest only. However, effects were seen in the differential nonuniformity (DNL), even though the ADC was not biased during the irradiation. Figure 13 shows a plot of the DNL. It is seen that there are missing codes for the low order bits. In effect the ADC is functioning as a 9-bit device.

Responsivity values were obtained for with a tungstenhalogen lamp calibrated against a UDT S390 radiometer. Measurements were made using narrow $(10 \mathrm{~nm})$ interference filters and for broadband (no filter) illumination. The relative ratios between the 5.3 Mrd device and the means for unirradiated devices are given below:

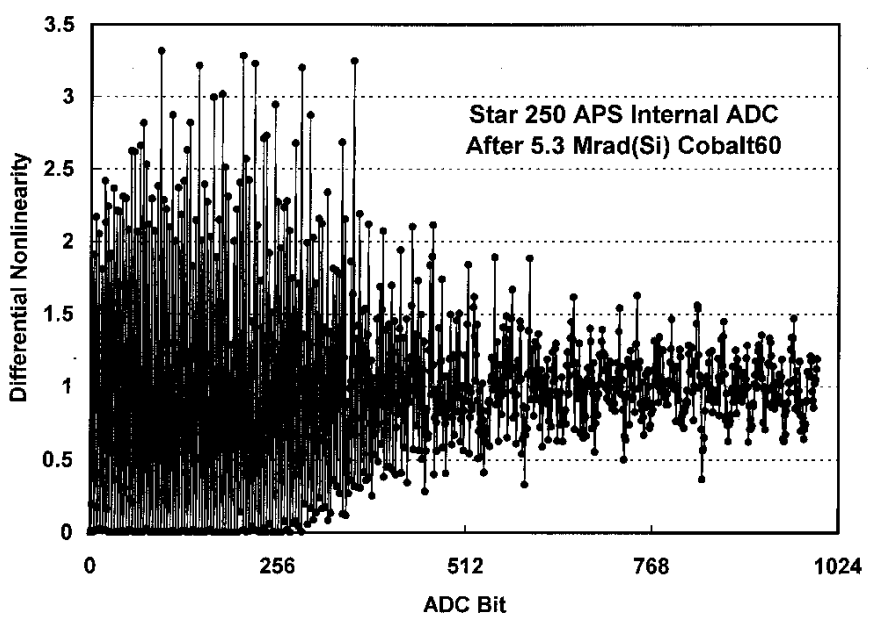

Fig 13 Differential nonlinearity for the on-chip 10-bit ADC. This is not used in the camera and was not biased during the irradiation. Unirradiated devices showed a uniform DNL (with the behavior above bit 512 shown for the lower bits also).

$\begin{array}{ll}\text { Broadband Illumination (BBI) } & 0.58 \\ \text { BBI + near IR cutoff filter } & 0.72 \\ 540 \mathrm{~nm} \text { filter } & 1.7 \\ 650 \mathrm{~nm} \text { filter } & 1.5 \\ 810 \mathrm{~nm} \text { filter } & 1.6\end{array}$

The spread in the average responsivity values for the unirradiated devices (including measurement errors) was $\pm 15 \%$ so it is seen that the departures from unity relative responsivity are probably significant. The irradiated APS seems to have increased responsivity in the range $540-810 \mathrm{~nm}$ but decreased responsivity for broadband illumination which contains the above range plus wavelengths in the blue. The reason for the changes is unknown at present and further investigations are planned.

\section{SUMMARY}

The results of cobalt60 irradiation show that CMOS active pixel sensor cameras can be used for the type of high total dose application, up till now reserved primarily for tube based cameras and systems based on photographic film. This paves the way for the more accurate metrology, ease of image manipulation and speed of data capture which are already well appreciated in conventional digital cameras. The APS is a low cost, off-the-shelf component and the camera is of simple design, requiring no bias voltage adjustments during irradiation. This is possible because of the low threshold voltage shift of the star-250 chip after irradiation. The ability to use only a minimal number of external electronic components in the camera head (for biasing and video buffering) has also been demonstrated and this leads to a miniaturized design (and also a low recurrent cost).

Laboratory tests performed 5.5 months after the irradiation have quantified the effects on the APS image sensor (at room temperature). Although there were noticeable changes in dark current and responsivity, these are not large enough to prevent use for TV applications after 5 Mrd. Changes in the other parameters were negligible. The clock threshold voltage shift was only $80 \pm 20 \mathrm{mV}$.

Although this paper has concentrated on imaging applications for the nuclear industry, the star-250 CMOS APS is an excellent candidate for use in space systems since unlike a CCD, the device does not suffer from proton-induced charge transfer effects. For example, the device is to be used in a telescope alignment monitor for NASA's SWIFT X-ray astronomy mission (the flight model was delivered in January 2002) and is being designed into equipment for inter-satellite laser communications (where the radiation tolerance and fast windowed readout are particularly advantageous). A program of total dose, proton and heavy-ion irradiation for space applications is underway and will be reported in a separate paper at a later date. 


\section{ACKNOWLEDGMENT}

The irradiations and test measurements described here were arranged by Magnox Electric plc and Tony August is thanked for his considerable help and support. The irradiations and dosimetry were performed by Keith Lovell of Cranfield University.

\section{REFERENCES}

[1] E.-S. Eid, T. Y. Chan, E. R. Fossum, R. H. Tsai, R. Spagnuoto, J. Deily, W. B. Byers Jr., and J. C. Peden, "Design and characterization of ionizing radiation-tolerant CMOS APS image sensors up to $30 \mathrm{Mrd}(\mathrm{Si})$ total dose", IEEE Trans. on Nucl. Sci., vol. 48, no. 6, pp. 1796-1806, Dec 2001.

[2] J. Carbone, S. Czebiniak and R. Carta "New CID detectors/cameras for use in ionizing radiation environments", Proc. 43rd Conference on Robotics and remote Systems, pp 43-50.

[3] J. Carbone, J. Zarnowski, M. Pace, S. Czebiniak and R. Carta, "Megarad and scientific CIDs", Proc SPIE, vol. 2028, pp. 131-138, 1996.

[4] On-line information available on http://www.fillfactory.com $/ \mathrm{htm} / \mathrm{cmos} / \mathrm{htm} / \mathrm{star} . \mathrm{htm}$

[5] G. R. Hopkinson, "Radiation Effects in a CMOS Active Pixel Sensor", IEEE Trans. on Nucl. Sci., vol. 47, no. 6, pp. 2480-2484, Dec 2000.

[6] J. Bogaerts and B. Dierickx, "Total dose effects on CMOS active pixel sensors", Proc SPIE, vol.3965, pp 157-167, January 2000. 